The most recent UK Retinopathy of Prematurity Guideline was introduced in May 2008. ${ }^{4}$ All babies born less than 32 weeks gestational age (GA) (up to 31 weeks and 6 days) or less than $1501 \mathrm{~g}$ birth weight should be screened for ROP. Infants born before 27 weeks GA (ie up to 26 weeks and 6 days) should have their initial ROP screening examination at $30-31$ weeks postmenstrual age (PMA).

We reviewed the screening records of all premature babies born prior to 27 weeks ( 26 weeks and 6 days) GA and subsequently screened at 30 weeks (up to 30 weeks and 6 days) in a single unit in Edinburgh between February 2005 and February 2015. Sixty-eight infants were identified.

Forty-four of the examined infants had tunica vasculosa lentis/persistent fetal vasculature, which limited the fundal view. Five screening examinations had to be postponed owing to absence of any fundal view.

Three babies had ROP. Two had Zone II Stage 1 with no plus disease and one had Zone II Stage 3 with no plus disease. Treatment was not required for any infant at the time of this initial examination. All infants were followed up within a 1 to 2 week interval until they met the termination of ROP screening criteria or developed ROP and received treatment; according to the criteria set out in the guidelines.

We suggest that the UK screening guideline could be revised to a first ROP screening examination for premature babies born prior to 27 weeks to be performed at 31 weeks ( 31 weeks and zero days to 31 weeks and 6 days) PMA. This revision would align the UK screening protocol with the current American Academy of Pediatrics/American Academy of Ophthalmology/ American Association of Pediatric Ophthalmology and Strabismus recommendation for 22-27 weeks GA infants to initiate screening at 31 PMA. ${ }^{5}$ Data from other neonatal units in the UK are needed to add confidence to this suggestion.

This change would allow premature babies an additional week before their first examination to stabilise without compromising their safety.

\section{Conflict of interest}

The authors declare no conflict of interest.

\section{References}

1 Rahi JS, Cable N, British Childhood Visual Impairment Study Group. Severe visual impairment and blindness in children in the UK. Lancet 2003; 362(9393): 1359-1365.

2 Laws DE, Morton C, Weindling M, Clark D. Systemic effects of screening for retinopathy of prematurity. $\mathrm{Br} J$ Ophthalmol 1996; 80(5): 425-428.

3 Rush R, Rush S, Nicolau J, Chapman K, Naqvi M. Systemic manifestations in response to mydriasis and physical examination during screening for retinopathy of prematurity. Retina 2004; 24(2): 242-245.

4 Wilkinson AR, Haines L, Head K, Fielder AR. UK retinopathy of prematurity guideline. Eye 2008; 23(11): 2137-2139.

5 Fierson WM, American Academy of Pediatrics Section on Ophthalmology, American Academy of Ophthalmology, American Association for Pediatric Ophthalmology and Strabismus, American Association of Certified Orthoptists.
Screening examination of premature infants for retinopathy of prematurity. Pediatrics 2013; 131(1): 189-195.

G Kontos ${ }^{1}$, A Khan ${ }^{2}$ and BW Fleck ${ }^{2}$

${ }^{1}$ Royal Glamorgan Hospital (RGH), Ynysmaerdy, Pontyclun, Mid Glamorgan, Wales, UK

${ }^{2}$ The Princess Alexandra Eye Pavilion (PAEP),

Edinburgh, Scotland, UK

E-mail: georgios.kontos@nhs.net

Eye (2016) 30, 636-637; doi:10.1038/eye.2015.279; published online 22 January 2016

Sir,

Treat and extend regimen with aflibercept for choroidal neovascularization in angioid streaks

Choroidal neovascularization (CNV) secondary to angioid streaks (AS) is a cause of important vision loss. The use of antivascular endothelial growth factors to treat CNV due to AS is well described. Until now, only experience with bevacizumab and ranibizumab had been reported to be an effective option of treatment. ${ }^{1}$ Recently, Vaz-Pereira et al reported in Eye the first two cases of $\mathrm{CNV}$ due to AS in which intravitreal aflibercept was used as primary treatment with pro re nata (PRN) regimen. ${ }^{2}$ We suggest the option of treat and extend regimen for this type of cases. We present the first case of CNV in AS in which intravitreal aflibercept was used following a treat and extend regimen with a follow-up period of 1 year.

\section{Case report}

A 60-year-old woman came to the emergency room with metamorphopsia in her left eye (LE). She had the medical antecedent of CNV secondary to AS in her right eye (RE) treated with 11 intravitreal injections of bevacizumab following a PRN regimen and a bad evolution. A complete ophthalmic examination was done. Her best corrected visual acuity (BCVA) was 20/200 in RE and 20/25 in LE. The anterior pole did not show any abnormality. The intraocular pressure was 15 and $17 \mathrm{mmHg}$, respectively. Funduscopy showed a disciform membrane in RE and AS in both eyes. Optical coherence tomography (OCT) revealed subretinal fibrosis with distortion of the foveal anatomy in RE and an incipient CNV in LE (Figure 1) confirmed by fluorescein angiography. Intravitreal aflibercept was injected in LE, one injection a month during 3 months. BCVA in LE improved to 20/20 and also OCT. After the good response in LE and bad evolution in RE, a treat and extend regimen was followed in LE. After 1-year period of follow-up, the BCVA is 20/20 in LE with no activity of CNV after six intravitreal aflibercept injections (Figure 2). 

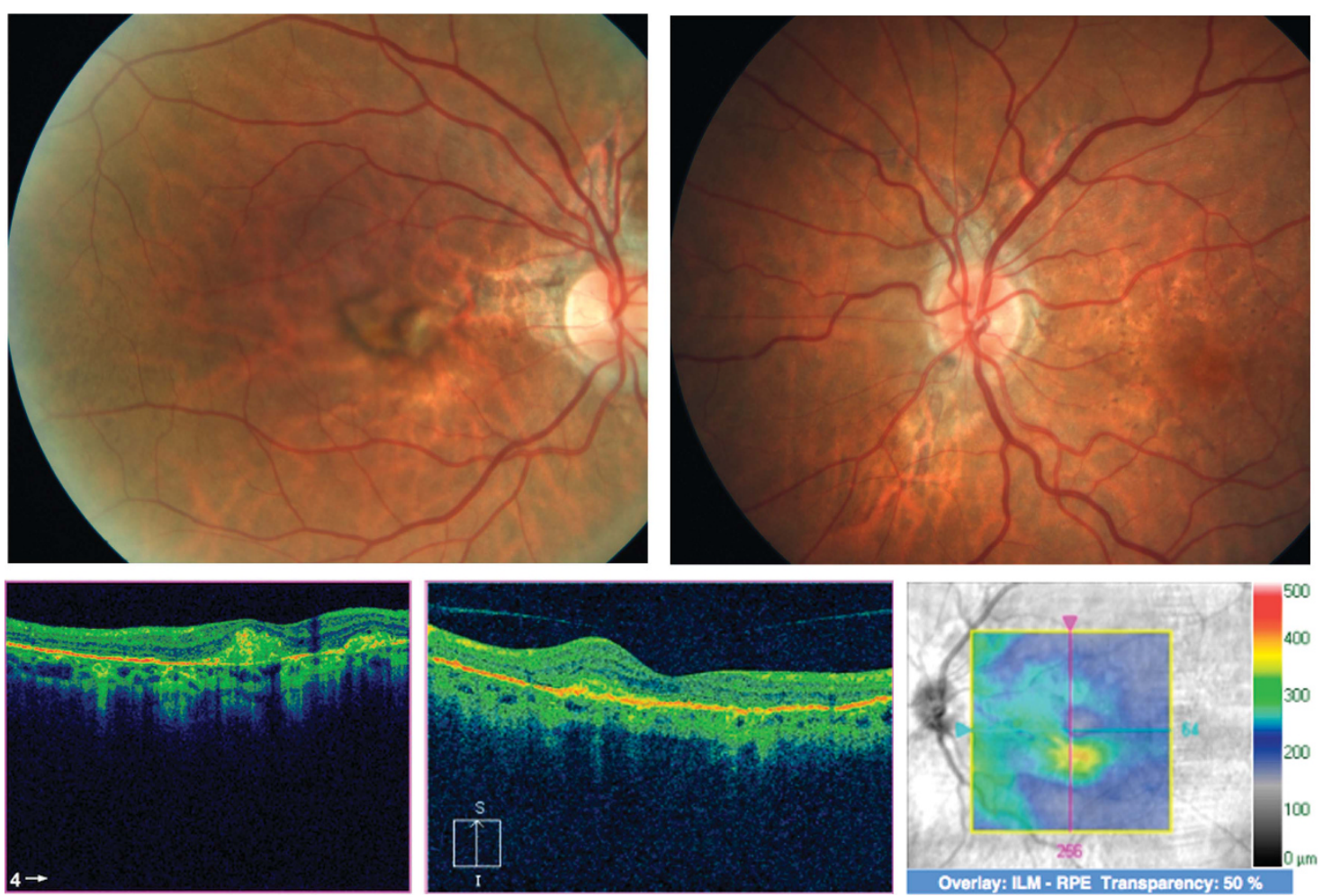

Figure 1 Funduscopy of RE and LE in which AS can be observed; macular OCT of RE and LE at the moment of the diagnosis of incipient CNV in LE.
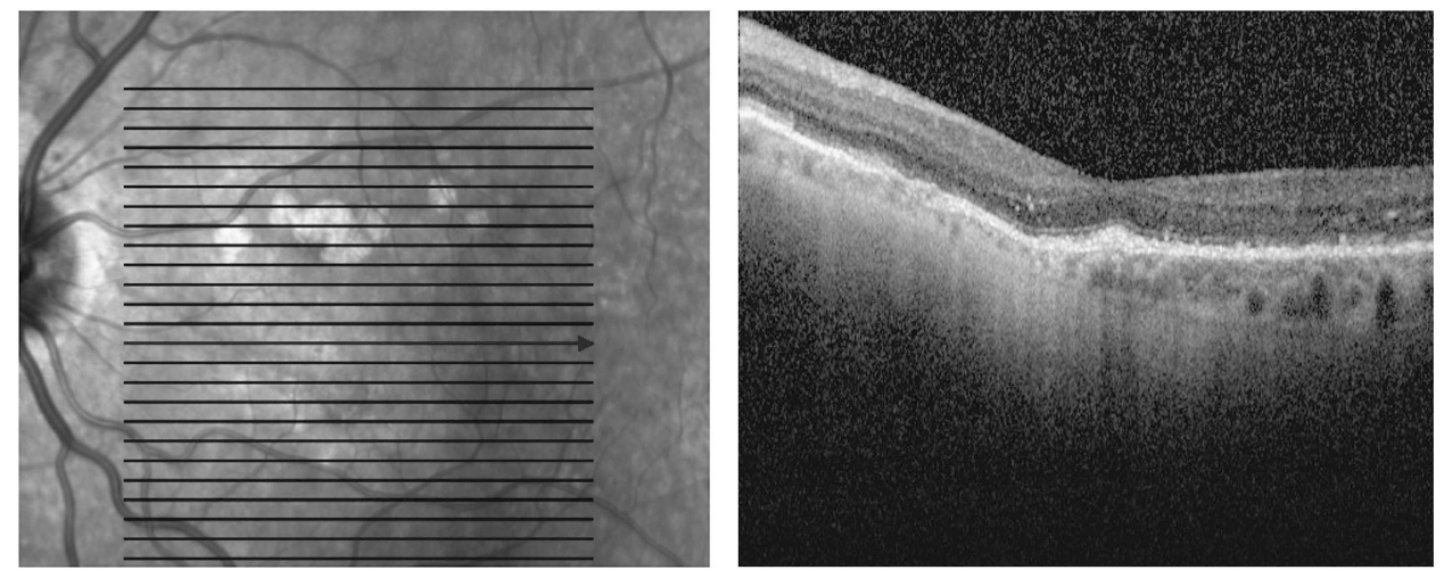

Figure 2 Retinography with macular OCT of LE after 12 months of treatment with intravitreal aflibercept following a treat and extend regimen.

\section{Comment}

The use of intravitreal aflibercept in a treat and extend regimen may be a good option for the management of CNV due to AS. Further prospective studies on a larger number of patients are required.

\section{Conflict of interest}

The author declares no conflict of interest.

\section{Acknowledgements}

Dr Diago has received travel support from Allergan, Novartis, and Bayer.

\section{References}

1 Esen E, Sizmaz S, Demircan N. Intravitreal aflibercept for management of subfoveal choroidal neovascularization 
secondary to angioid streaks. Indian J Ophthalmol 2015; 63(7): 616-618

2 Vaz Pereira S, Collaco L, De Salvo G, van Zeller P. Intravitreal aflibercept for choroidal neovascularisation in angioid streaks. Eye 2015; 29(9): 1236-1238.

\section{T Diago}

Department of Ophthalmology, Unit of Macula, Hospital Universitario de La Ribera, Alzira, Valencia, Spain

E-mail: tediasem@gmail.com

Eye (2016) 30, 637-639; doi:10.1038/eye.2015.283; published online 22 January 2016

\section{Sir, Intravitreal aflibercept for choroidal neovascularisation in angioid streaks}

We thank Diago ${ }^{1}$ for the interest shown in our recent article. We reported for the first time the use of intravitreal aflibercept as primary treatment for choroidal neovascularisation (CNV) in angioid streaks (AS) using a pro re nata regimen. ${ }^{2}$ This approach, in which treatment is withheld unless there is CNV activity, has been the most widely adopted in this setting. 3,4

Treat and extend (T\&E) is a treatment modality that aims to treat the CNV proactively, in order to find an optimal treatment interval, and has been more commonly used in the context of neovascular age-related macular degeneration (nAMD). While we find the T\&E modality to be an interesting approach for AS-associated CNV, we agree with previous reports that the CNV in this context is closer to myopia-related $\mathrm{CNV}$ than nAMD-related $\mathrm{CNV}$, and therefore may require fewer injections. ${ }^{4}$ Additionally, patients can be activity-free for several years, or develop new CNV lesions in a different location altogether, making it difficult to identify a specific pattern of recurrence. ${ }^{4}$ This could lead to overtreatment with subsequent increase of associated risks such as chorioretinal atrophy. ${ }^{5}$

Even though larger prospective trials are needed to elucidate on the best treatment protocol, they are difficult to arrange due to the rarity of the condition.

\section{Conflict of interest}

Dr Vaz-Pereira has received consultant fees from Bayer and Novartis and has received travel grants from Bayer, Novartis, Alcon, Allergan and Alimera Sciences. Dr Collaço has received travel grants from Bayer. Dr De Salvo has received travel grants from Bayer and Heidelberg Engineering. Dr van Zeller declares no conflict of interest.

\section{References}

1 Diago T. Treat and extend regimen with aflibercept for choroidal neovascularization in angioid streaks. Eye (Lond) 2016; 30: 637-639.
2 Vaz-Pereira S, Collaço L, De Salvo G, van Zeller P. Intravitreal aflibercept for choroidal neovascularisation in angioid streaks. Eye (Lond) 2015; 29: 1236-1238.

3 Gliem M, Finger RP, Fimmers R, Brinkmann CK, Holz FG, Charbel Issa P. Treatment of choroidal neovascularization due to angioid streaks: a comprehensive review. Retina 2013; 33: 1300-1314.

4 Tilleul J, Mimoun G, Querques G, Puche N, Zerbib J, Lalloum $\mathrm{F}$ et al. Intravitreal ranibizumab for choroida neovascularization in angioid streaks: four-year follow-up. Retina 2015; e-pub ahead of print 9 September 2015.

5 Grunwald JE, Daniel E, Huang J, Ying GS, Maguire MG, Toth CA et al. CATT Research Group. Risk of geographic atrophy in the comparison of age-related macular degeneration treatments trials. Ophthalmology 2014; 121: 150-161.

S Vaz-Pereira ${ }^{1,2,3}$, L Collaço $^{1}, \mathrm{G}$ De Salvo ${ }^{4}$ and P van Zeller ${ }^{1}$

1Department of Ophthalmology, Clínica São João de Deus, Lisbon, Portugal

${ }^{2}$ Department of Ophthalmology, Hospital de Santa

Maria, Lisbon, Portugal

${ }^{3}$ Department of Ophthalmology, Faculty of Medicine,

University of Lisbon, Lisbon, Portugal

${ }^{4}$ Eye Unit, University Hospital Southampton NHS

Foundation Trust, Southampton, UK

E-mail: saravazpereira@gmail.com

Eye (2016) 30, 639; doi:10.1038/eye.2015.284;

published online 22 January 2016

Sir,

A rapidly emerging ocular zoonosis; Dirofilaria repens

Dirofilariasis—commonly caused by Dirofilariasis repens and $D$. immitis and transmitted via mosquito bites-is a roundworm zoonosis that is emerging as a public health concern in Eastern and Southern Europe, Asia Minor, Central Asia, and Sri Lanka, possibly due to climatic changes. ${ }^{1,2}$ Until 1999, only a single case of dirofilariasis was reported from Turkey; however, the number of reported cases in Turkey increased to 22 between 1999 and 2011. Here we report three new cases of $D$. repens detected in Marmara Region of Turkey, which were witnessed in June 2013 with accompanying history of mosquito bites and lack of abroad travelling.

\section{Case report}

The first case-56-year-old female patient-suffered presumed viral conjunctivitis on both eyes since 3 weeks, for which she received symptomatic and topical steroid treatment. The infectious condition on her left eye got worse during last few days before her recent evaluation. Slit-lamp biomicroscopic examination revealed a new convoluted, translucent, $6 \times 8 \mathrm{~mm}$-sized, immobile, and subconjunctival larva (Figure 1). Second 31-year-old female patient presented with the complaint of stinging in her left eye; a superotemporal, $3 \times 2$-mm-sized, convoluted, translusent, and immobile subconjunctival larva was detected with accompanying episcleritis. The third 63-year-old male patient had admitted for routine 